\title{
Endoscopic management of biliobronchial fistulas
}

\begin{abstract}
Bronchobiliary fistula (BBF), which often presents with bilioptysis, is an abnormal communication between the bronchial system and biliary tree. It is a complication associated with a high mortality rate and requires a well-planned management strategy. Although hydatid disease is still the leading cause, extensive surgical interventions and invasive procedures of the liver have altered the profile of patients in recent decades. This paper presents 5 cases of BBF and reviews the literature regarding the treatment options generally mandated by clinical presentation and the underlying disease.
\end{abstract}

Objective: To determine the effect of endoscopic sphincterotomy in the management of Bronchobiliary fistula.

Design: A case study between January 2011 and December 2016.

Setting: Department of Visceral Surgery, Hassan II University Hospital, Fez, Morocco

Patients: Five patients with hepatic hydatid cyst, in which the cyst had ruptured into the bronchial tree and the biliary channels.

The follow-up ranged from 7 to 36 months.

Intervention: Endoscopic sphincterotomy.

Main outcome measures: Morbidity, mortality and recurrence of the disease

Results: All patients underwent successful endoscopic sphincterotomy, including removal of daughtercysts. During the follow-up period, ultrasonography and laboratory investigations showed complete cure in all patients. There were no complications due to endoscopic sphincterotomy.

Conclusion: Endoscopic sphincterotomy is the treatment of choice for the management of hydatid cysts that have ruptured into the bronchial tree and the biliary tract.

Keywords: bronchobiliary fistula; bilioptysis; hydatid cyst; endoscopic sphincterotomy
Volume 4 Issue $6-2017$

\author{
Alila M, Bourakkadi Idrissi M, Dkhissi Y, \\ Benjelloun E \\ Department of Visceral Surgery, Hassan II University Hospital, \\ Morocco
}
Correspondence: Mohammed Alila, Resident post-graduate in general surgery, Department of Visceral Surgery, Hassan II University Hospital, Fez, Morocco, Tel +212 662632625 , Emailmohammed.alila@gmail.com

Received: October 29, 2017 | Published: December 12, 2017
Abbreviations: $\mathrm{BBC}$, bronchobiliary fistula; ERCP, endoscopic retrograde cholangiopancreatography; CT Scan, computed tomography scan

\section{Introduction}

Bronchobiliary fistula (BBF), which requires aggressive treatment to decrease the mortality and morbidity rates, is an abnormal communication between the biliary channels and the bronchial tree. It occurs as a congenital malformation, but most patients have a history of liver pathology that involves the lungs. ${ }^{1}$ In the literature, the number of reported cases of BBF has increased significantly in recent years; however, management of this complication has not been extensively discussed. In this paper, we report 5 cases and review the literature regarding the endoscopic management of BBF.

\section{Patients and methods}

Five patients (four men and one woman) with a hydatid liver cyst that had ruptured simultaneously into the biliary and bronchial tree were investigated at the Department of Visceral Surgery, Hassan II University Hospital, Fez, Morocco, between January 2011 and December 2016. The patients ranged in age from 21 to 78 years (mean 43, 6 years). All patients presented with irritating cough, expectoration of yellowish sputum and cholestatic jaundice (Table 1). There was no history of jaundice or biliptysis in any of the patients. The liver cyst had ruptured spontaneously in all patients. Complete blood count, liver function tests, abdominal ultrasonography, computed tomography and ERCP were carried out in all patients (Table 1). The indirect hemagglutination test was also performed to determine the titre for hydatid disease. The disease was managed endoscopically in all patients. The treatment included endoscopic sphincterotomy and extraction of daughter cysts or hydatid membranes from the biliary tree. After the endoscopic procedure all patients underwent a cyst dome resection by laparotomy and received mebendazole. The follow-up ranged from 7 to 36 months, and consisted in a thoracoabdominal CT scan, ultrasonography and laboratory investigations.

\section{Results}

On admission all five patients had an elevated leukocyte count (11.0 to $37.0 \mathrm{~d} 10 / \mathrm{L}$ ). Two patients had eosinophilia (over $5 \%$ ). Liver function test results were abnormal in all patients; they had elevated levels of bilirubin, aminotransferases, alkaline phosphatase and $\gamma$-glutamyl transpeptidase. The indirect hemagglutination titre for hydatid disease was elevated in all patients (range from 1:412 to 1:1926). Abdominal ultrasonography demonstrated a hydatid liver cyst and different degrees of dilatation of the bile ducts. The CT scan showed a cystic image of the liver appearing to communicate with a pulmonary lesion in two out of our five cases (Figure 1) with patchy densities over the right lung for four patients and for the fifth revealed that the volume of the right hemithorax was diminished with homogeneous density at the basal segments. Cholangiopancreatography endoscopic retrograde 
(ERCP) revealed dilatation of the biliary tree, communicating with the hydatid liver cysts and helped visualize the Biliobronchial fistula in all patients. Endoscopic sphincterotomy was carried out in all cases. In all our cases, hydatid daughter cysts were impacted in the ampulla of Vater. All patients underwent successful endoscopic sphincterotomy, including removal of daughtercysts (Figure 2). No complications due to endoscopic procedures occurred. After the endoscopic procedure all patients underwent a cyst dome resection, suture of the diaphragmatic defect after hepatodiaphragmatic disconnection by laparotomy.

Table I Clinic and radiologic finding in five patients with Biliobronchial fistula due to hydatid cyst

\begin{tabular}{|c|c|c|c|}
\hline Case No & Age, yr/ Sex & Presentation & Ultrasonographic and CT findings \\
\hline I & $47 / F$ & $\begin{array}{l}\text { Obstructive jaundice massive biliptysis } \\
\text { irritating cough right thoracoabdominal } \\
\text { pain. Hemoptysis Fever }\end{array}$ & $\begin{array}{l}6 \mathrm{~cm} \text { hydatid cyst of the hepatic dome Dilated intrahepatic biliary tree } \\
\text { and Common bile duct Evidence BBF Density at the basal of right lung }\end{array}$ \\
\hline 2 & $21 / M$ & cough, dyspnea, yellow green sputum & hepatic hydatid cyst $(8 \mathrm{~cm})$ patchy densities over the right lung \\
\hline 3 & $43 / \mathrm{M}$ & Obstructive jaundice biliptysis & $9 \mathrm{~cm}$ hydatid cyst of the hepatic dome Density at the basal of right lung \\
\hline 4 & 29/M & $\begin{array}{l}\text { Obstructive jaundice Biliptysis right } \\
\text { thoracoabdominal pain Hemoptysis }\end{array}$ & $\begin{array}{l}\mathrm{I} \mathrm{Icm} \text { hydatid cyst of the hepatic dome. pulmonary crackin with } \\
\text { evidence of a fistulous pathway homogeneous density at the basal of } \\
\text { right lung }\end{array}$ \\
\hline 5 & $78 / \mathrm{M}$ & $\begin{array}{l}\text { Obstructive jaundice Bilioptysis dyspnea, } \\
\text { right thoracoabdominal pain }\end{array}$ & a multiple hepatic hydatid cyst patchy densities over the right lung \\
\hline
\end{tabular}

M, male; F, female

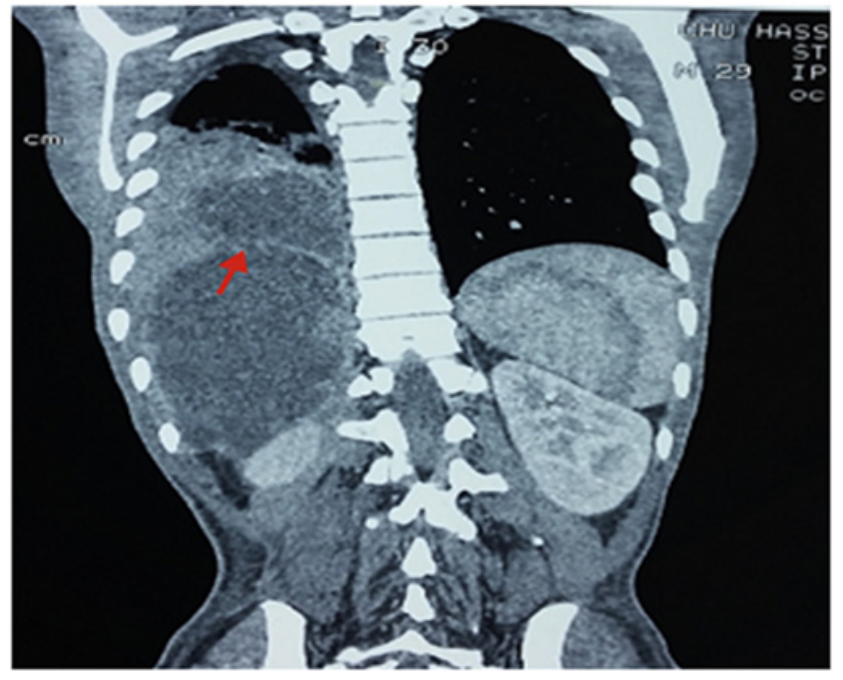

Figure I Thoraco-abdominal computed tomography showing a hydatid cyst of the hepatic dome ruptured in the lung.

In the case of one out of our five reported patients, right thoracotomy was performed for a third time. The lower lobe was almost totally damaged, and dissection between the liver and lung was extremely difficult. Therefore, we decided to perform lobectomy, and the lobe was resected along with a part of the diaphragm. The dome of the liver was closed with capitonage, and the diaphragm was closed primarily after inserting a subcostal drain. The patient was discharged on day 5 after the drains were removed. Subsequently, all patients received mebendazole (400mg twice daily) for 3 weeks followed by a one -week drug-free period for six courses. No side effects of mebendazole were noted. Ultrasonography and thoracoabdominal CT scan were repeated during the follow-up period, which ranged from 3 to 36 months. No morbidity, mortality or recurrence of the disease were noted.

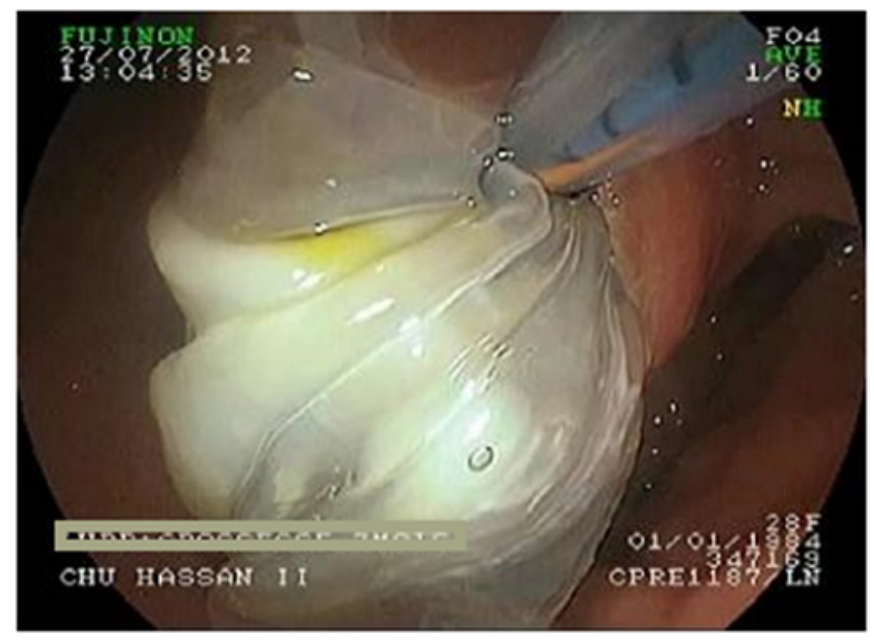

Figure 2 Endoscopic view after sphincterotomy with extraction of Hydatid membranes.

\section{Discussion}

Bronchobiliary fistula (BBF) is defined as an abnormal communication between the biliary system and bronchial tree. In 1850 , Peacock described the first case of BBF in a patient presenting with liver hydatidosis. ${ }^{2} \mathrm{BBF}$ has also been reported as a congenital malformation; however, in most cases, it occurs following liver pathology, particularly parasitic infections. Several mechanisms are suggested for the development of BBF, including an inflammatory reaction in the sub diaphragmatic space with subsequent ruptures into the bronchial system and liver pathology which erodes the diaphragm leading a communication between bronchial tree and biliary channels. Although hydatid disease is still the leading cause of BBF. Apart from anecdotal series that comprise a large number of cases, ${ }^{3,4}$ studies dealing with BBF rarely report more than a few cases. BBF is a serious 
complication associated with a high mortality and morbidity rate $(12.2 \%)^{5}$ and requires a well-planned management strategy. Despite the increasing number of reported cases, a widely accepted strategy for the management of BBF remains to be defined.

Bile behaves as a strong irritant when present outside the biliary channel and gastrointestinal system. ${ }^{6}$ Regarding the circumstances of the discovery, the only specific sign is biliptysis, which may be more or less abundant. Sometimes bronchopulmonary infection may be the main aspect of the clinical symptomatology often associated with non specific signs such as cough and thoracoabdominal pain. Thus, the diagnosis of $\mathrm{BBF}$ of hydatid origin must be based on clusters of clinical arguments, documented by para-clinical and radiological examinations. ${ }^{2,6,7}$ Radiology reveals patch densities dominantly in the right lower lobe; however, these patches may also be present throughout the right lung and even in the left side.,4,6 Thoracic and upper abdominal CTs appear to be the best tools for the initial evaluation of lung and liver pathologies to plan further investigations, the presence of a cystic image of the liver appearing to continue a pulmonary lesion that is variable in a patient with biliptysis in a hydatid endemic area is an incontestable the diagnosis.

Hepatobiliary ultrasound should seek an obstacle and or dilation of the main bile duct. ${ }^{8}$ It also allows a better examination the hydatid cyst of the liver, specifying its type and its exact localization in the hepatic segments. ${ }^{9}$ The search for biliary pigments and scolexes in sputum or bronchial fibro-aspiration fluid must be systematic. It is a simple examination which gives a diagnosis of certainty in the absence of obvious biliptysis. Until recently, surgical removal of liver and biliary hydatid cysts was the only form of therapy, but this surgical procedure is associated with numerous complications and treatment of Bronchobiliary fistula fails if the obstruction in the biliary channel persists. Therefore, the first step after diagnosis is ensuring unobstructed bile drainage into the duodenum, which will decrease the bile leakage. Management of a bronchobiliary fistula without biliary obstruction includes surgery with thoracotomy or a thoracoabdominal approach. ${ }^{2,6}$

Retrograde endoscopic cholangiography is an important step in the preparation for surgery. It allows the visualization of the fistula and look s for a possible obstruction of the bile duct. ${ }^{10}$ The addition to the sphincterotomy increases its profitability by allowing the extraction of hydatid membrane or the placement of prosthesis of the bile duct in order to establish a normal biliary current thus minimizing the risk of recurrence after the surgical treatment. This very interesting examination can be carried out in preoperative surgery by a biliary cartography to look for a possible obstacle of the main bile duct of which its ignorance can condition the surgeries and the failure of treatment. When using laparotomy, the treatment of hepatic lesions is only possible after the liberation of the hepaticdome of the diaphragm; it consists of treating the hepatic residual cavity and the biliary fistula after a most often partial resection of the cystic wall (cyst dome resection) followed by closure of the biliary fistula. ${ }^{2,6}$ We must insist on the treatment of the Bronchobiliary fistula. Rarely is it necessary to carry out an anatomic lung resection (segmentectomy, lobectomy) for important lesions of the lung parenchyma which requires a complementary thoracotomy.

\section{Conclusion}

Bronchobiliary fistulas due to hydatid cyst are rare, but severe disease. A high perioperative mortality rate has been observed historically. Endoscopic management of this disease is an effective, safe method and prevents serious complications. We believe that the aforementioned surgical approach may be the treatment of choice in the management of cases of biliobronchial fistula.

\section{Acknowledgements}

Mohammed Alila, wrote the paper and gathered referenced data. Mehdi Bourakkadi Idrissi, Yassin Dkhissi: gathered referenced data, contributed equally in organizing them, and reviewed the draft. E. Benjelloun, reviewed the final paper.

\section{Conflicts of interest}

The authors had no conflict of interest to declare.

\section{References}

1. Singh B, Moodley J, Sheik-Gafoor MH, et al. Conservative management of thoracobiliary fistula. Ann Thorac Surg. 2002;73(4):1088-1091.

2. Msaad S, Yangui I, Ketata W, et al. Hydatid cysts of the liver ruptured into the thorax (about five cases). Rev Pneumol Clin. 2015;75(5):255263.

3. Gerazounis M, Athanassiadi K, Metaxas E, et al. Bronchobiliary fistulae due to echinococcosis. Eu J Cardiothorac Surg. 2002;22(2):306-308.

4. Kabiri H, Maslout AE, Benosman A. Thoracic rupture of hepatic hydatidosis (123 cases). Ann Thorac Surg. 2001;72(6):1883-1886.

5. Moumen M, El Fares F. Biliobronchial fistula of hydatid origin. Apropos of 8 cases. J Chir (Paris). 1991;128(4):188-192.

6. Chua HK, Allen MS, Deschamps C, et al. Bronchobiliary fistula: principles of management. Ann Thorac Surg. 2000;70(4):1392-1394.

7. Khandelwal M, Inverso N, Center R, et al. Endoscopic management of a bronchobiliary fistula. J Clin Gastroenterol. 1996;23(2):125-127.

8. Moumen M, El Fares F. Biliobronchial fistulas of hydatid origin. Apropos of 8 cases. J Chir. 1961;128(4):188-192.

9. Gharbi HA, Hassine W, Brauner MW, et al. Ultrasound examination of the hydatic liver. Radiology. 1981;139(2):459-463.

10. Dziri C, Haouet K, Fingerhut A, et al. Management of cystic echinococcosis complications and dissemination: where is the evidence? World J Surg. 2009;33(6):1266-1273. 